\title{
Tangent function transformation of the Abbreviated Injury Scale improves accuracy and simplifies scoring
}

Muding Wang ${ }^{1}$, Wusi Qiu ${ }^{2}$, Fang Qiu ${ }^{1}$, Yinan $\mathrm{Mo}^{3}$, Wenhui Fan ${ }^{1}$

\author{
'Department of Emergency Medicine, Affiliated Hospital of Hangzhou Normal \\ University, Hangzhou, China \\ 2Department of Neurosurgery, Hangzhou Second Hospital, College of Medicine, \\ Hangzhou Normal University, Hangzhou, China \\ ${ }^{3} 3^{\text {rd }}$ Ward of Hand Surgery, Fengtian Hospital affiliated to Shenyang Medical College, \\ Shenyang, China
}

Submitted: 3 January 2013

Accepted: 20 February 2013

Arch Med Sci 2015; 11, 1: 130-136

DOI: $10.5114 /$ aoms.2015.49209

Copyright $\odot 2015$ Termedia \& Banach

\section{Abstract}

Introduction: The Injury Severity Score (ISS) and the New Injury Severity Score (NISS) are widely used for anatomic severity assessments after trauma. We present here the Tangent Injury Severity Score (TISS), which transforms the Abbreviated Injury Scale (AIS) as a predictor of mortality.

Material and methods: The TISS is defined as the sum of the tangent function of AIS/6 to the power 3.04 multiplied by 18.67 of a patient's three most severe AIS injuries regardless of body regions. TISS values were calculated for every patient in two large independent data sets: 3,908 and 4,171 patients treated during a 6-year period at level-3 first-class comprehensive hospitals: the Affiliated Hospital of Hangzhou Normal University and Fengtian Hospital Affiliated to Shenyang Medical College, China. The power of TISS to predict mortality was compared with previously calculated NISS values for the same patients in each data set.

Results: The TISS is more predictive of survival than NISS (Hangzhou: receiver operating characteristic $(\mathrm{ROC})$ : NISS $=0.929$, TISS $=0.949 ; p=0.002$; Shenyang: ROC: NISS $=0.924$, TISS $=0.942 ; p=0.008)$. Moreover, TISS provides a better fit throughout its entire range of prediction (Hosmer Lemeshow statistic for Hangzhou NISS $=29.71 ; p<0.001$, TISS $=19.59 ; p=$ 0.003; Hosmer Lemeshow statistic for Shenyang NISS $=33.49 ; p<0.001$, TISS $=21.19 ; p=0.002$ )

Conclusions: The TISS shows more accurate prediction of prognosis and a linear relation to mortality. The TISS might be a better injury scoring tool with simple computation.

Key words: trauma, critical care, injury scale, history.

\section{Introduction}

The Abbreviated Injury Scale (AIS) is specifically designed as a system to define anatomically the severity of injuries throughout the body. It has been revised, expanded and improved repeatedly. Its recent revision was the AIS 2005 edition, update 2008 [1]. AIS codes are assigned to each of a patient's injuries and include the AIS severity measure, an ordinal value that ranges from 1 to 6 . Minor injuries have an AIS value of 1 , and injuries that are thought to be currently untreatable are given a 6 [2].

\author{
Corresponding author: \\ Prof. Wusi Qiu PhD \\ Department of Neurosurgery \\ Hangzhou Second Hospital \\ College of Medicine \\ Hangzhou Normal University \\ 126 Wenzhou Road \\ Gongshu District \\ 310015 Hangzhou (Zhejiang) \\ China \\ Phone: +86 13858019591 \\ E-mail: shihai954@163.com
}


Although the AIS provided a rudimentary dictionary of possible injuries, it failed to provide a mechanism to summarize a single patient's multiple injures into a single score. This step was taken by Baker et al. in 1974 with the creation of the Injury Severity Score (ISS) [3]. The ISS is used to describe trauma populations, to evaluate the quality of trauma care, and to control for case mix in trauma research [3]. It is based on AIS severity values, which are assigned from the patient's hospital files at discharge. The ISS reflects deterioration in patient status after trauma but does not discriminate between deterioration secondary to the natural history of the injury and in-hospital care. It therefore may not be a valid instrument for evaluating the quality of trauma care. Perhaps the most important drawback of the ISS cannot be addressed with statistical techniques: the ISS only considers one injury in each body region. This leads to injuries being overlooked and to less severe injuries occurring in other body regions being included in the calculation over more serious ones in the same body region. A simple modification to the ISS, the New Injury Severity Score (NISS), was designed by Osler et al. in 1997 to counter this problem [4]. The NISS is simply the sum of squares of the three most severe injuries, regardless of body regions injured.

Many articles existing in the literature have compared the NISS to the ISS in terms of mortality. Most of the studies have showed better discrimination and calibration for NISS [5-8], and some studies have observed better calibration but equivalent discrimination $[9,10]$. In addition, a few studies have noted no advantage for the NISS [11, 12]. Some studies [13-16] have revealed that the mortality associated with AIS severity combinations that produce identical ISS/NISS is different. ISS/NISS scores with a higher AIS value significantly increased the risk of mortality. They serve to identify specific areas in which the ability to predict survival may be flawed, yet previously not acknowledged.

We therefore tested a simple modification of AIS values, a score that we call the Tangent Injury Severity Score (TISS). The TISS is the sum of the tangent function of AIS/ 6 to the power 3.04 multiplied by 18.67 of a patient's three most severe (i.e. highest AIS) regardless of body regions. This study aims to investigate whether the TISS statistically outperforms the NISS in predicting in-hospital mortality. In addition, the study examines whether the mortality associated with AIS severity combinations that produce identical ISS/NISS is different.

\section{Material and methods}

This retrospective cohort study reviewed data from level-3 first-class comprehensive hospital at the Affiliated Hospital of Hangzhou Normal University and Fengtian Hospital affiliated to Shenyang Medical College, China for the period from January 1, 2006 to December 31, 2011. The trauma registry contains data for two totals of 10,625 and 12,960 patients hospitalized as a result of trauma. It includes a complete listing of each patient's injuries in the AIS lexicon (2005 revision). This study was approved by the institutional review boards of each institution.

The information collected includes demographic data, details on the injury, physiologic variables throughout the patient's course, outcome, complications, and performance indicators. Trauma registrars, all fully trained in data collection and coding, are responsible for data collection. Regular quality assurance meetings and chart review ensure consistency of data collection, input, and interpretation. Patients with isolated minor or moderate injuries (AIS 1 and 2) to a single body region and deaths on arrival were excluded from the study. The study populations therefore comprised 3,908 and 4,171 patients. These data sets enable us to test the performance of NISS and TISS.

The NISS was computed according to Osler et al. [4]. The TISS was computed as the simple change of AIS values by raising each AIS severity score (1-6) by the tangent function of AIS/ 6 to the power 3.04 multiplied by 18.67 and then summing the three most severe (i.e. highest AIS) regardless of body region.

Regarding the development of TISS score conversion, this study can be described as follows: First, it is based on the National Trauma Database (NTDB) [2] statistics of 181,707 patients who sustained a single injury. The relation between their AIS values and mortality showed a quadratic function relation. We propose a change of AIS values by raising each AIS severity score (1-6) to adapt them to the corresponding proportion. Second, TISS inherits the highest ISS/ NISS score (when AIS 5, ISS/NISS value for $5^{2}=$ 25) and adds the three most severe (i.e. highest AIS). Third, the AIS values (i.e. 3, 4, and 5) that may lead to death must be an integer quantity after transformation for facilitating calculation and memory. Fourth, AIS 3 is unchanged after transformation. Fifth, according to the characteristic of the tangent function, with the increase of radians from 0 to $\pi / 2$, the corresponding value is also increased. So this study takes AIS/6 as the radians. Therefore, this study sets $X$ as the common power index of $\tan (\mathrm{AIS} / 6), Y$ as the value after transforming AIS 4 , and Z as the multiplier. Application of logarithmic algorithm to calculate the common power index: $X=(\log (25 / 3)) /$ $\log (\tan (5 / 6) / \tan (3 / 6))=(\log (25 / Y)) / \log (\tan$ $(5 / 6) / \tan (4 / 6))=3.026$. So, $Y=9.05$. Adjusting 
Table I. Tangent function transformation of AIS codes

\begin{tabular}{|cccccc|}
\hline $\begin{array}{c}\text { AIS codes } \\
(\mathrm{A})\end{array}$ & $\begin{array}{c}\mathrm{A} / 6 \\
(\mathrm{~B})\end{array}$ & $\begin{array}{c}\text { Tan } \mathrm{B} \\
(\mathrm{C})\end{array}$ & $\begin{array}{c}\mathrm{C}^{3.04} \\
(\mathrm{D})\end{array}$ & $\begin{array}{c}\mathrm{D} \times 18.67 \\
(\mathrm{E})\end{array}$ & $\begin{array}{c}\text { Accurate to one } \\
\text { decimal place }(\mathrm{F})\end{array}$ \\
\hline 1 & 0.167 & 0.1682 & 0.0044 & 0.08 & 0.1 \\
\hline 2 & 0.333 & 0.3463 & 0.0398 & 0.74 & 0.7 \\
\hline 3 & 0.500 & 0.5463 & 0.1591 & 2.97 & 3.0 \\
\hline 4 & 0.667 & 0.7868 & 0.4825 & 9.01 & 9.0 \\
\hline 5 & 0.833 & 1.1008 & 1.3390 & 25.00 & 25.0 \\
\hline $6^{*}$ & 1.000 & 1.5574 & 3.8451 & 71.79 & 75.0 \\
\hline
\end{tabular}

Tan means tangent function. *AIS code is 6 , TISS value of automatic promotion for 75 .

$X=3.04$, then $Y=9.01 \approx 9.0$. Therefore, the multiplier $Z=25 /(\tan (5 / 6))^{3.04}=18.67$ (Table I). In order to adjust the AIS 2 that has an effect on the value, 0.4 must be deducted from every total. These values should be based on the arithmetic rules and rounded to integral quantities. The TISS value is the sum of three round numbers in the F column (Table I) after a modification of AIS codes. (Mathematical expression: TISS $=\left(\tan \left(A_{1} / 6\right)\right)^{3.04} \times$ $18.67+\left(\tan \left(A_{2} / 6\right)\right)^{3.04} \times 18.67+\left(\tan \left(A_{3} / 6\right)\right)^{3.04} \times$ $18.67-0.4$ (round numbers). $A_{1}, A_{2}$, and $A_{3}$ are the three most severe AIS codes.) By way of example, a patient has 5 injuries as follows: Head AIS 5 and 2, Chest AIS 4, Abdomen AIS 2 and Lower extremities AIS 2. The three highest AIS codes are 5 , 4 , and 2. Therefore, TISS $=(\tan (5 / 6))^{3.04} \times 18.67+$ $(\tan (4 / 6))^{3.04} \times 18.67+(\tan (2 / 6))^{3.04} \times 18.67-0.4$ $=25+9+0.7-0.4=34.3 \approx 34$. NISS $=5^{2}+4^{2}+2^{2}$ $=25+16+4=45$.

\section{Statistical analysis}

Comparisons between NISS and TISS were made using misclassification rates, receiver operating characteristic (ROC) curve analysis, and the Hosmer Lemeshow $(\mathrm{H}-\mathrm{L})$ goodness of fit statistics by univariate and multivariate logistic regression models. Misclassification rates were defined as the sum of false positives and false negatives divided by the total number of cases. A risk of death of $50 \%$ was used as the cutoff for misclassification. The cutoff severity scores were 34, 34 for the TISS and 48, 45 for the NISS, in Hangzhou and Shenyang, respectively. The area under the ROC curve was used to test sensitivity and specificity. The $\mathrm{H}$ - $\mathrm{L}$ statistic is a measures of how well calibrated a model is. The result is evaluated by a $\chi^{2}$ test. Data were statistically compared by Pearson's $\chi^{2}$ test for categorical variables and Wilcoxon one-sample test or Mann-Whitney $U$ test for continuous variables. Statistical analyses were performed using SPSS 18.0 for Windows. A $p$ value $<0.05$ was considered statistically significant.

\section{Results}

The patients' average age was 43.5 years (range: 23-64 years) and 45.4 years (range: $25-66$ years) and male $74.0 \%$ and $70.8 \%$ in Hangzhou and Shenyang, respectively, bluntly injured (approximately 93\%). Approximately $94 \%$ survived to hospital discharge. The most frequently insulted body regions were the head and lower extremities. The majority of patients were injured in motor vehicle collisions or falls. The most frequent AIS code was 3 (approximately 65\%).

The TISS values of a few patients (approximately $2 \%$ ) were equal to the corresponding NISS values. This result is expected, because all AIS values were 5 or 6 code. In divergent observations, the difference between TISS and NISS values ranged from 1 to 21 , with a median of 11.3 and 11.0 in Hangzhou and Shenyang, respectively. The median NISS and TISS values were 21.3 and 10.0 and 20.8 and 9.8 , respectively.

When we examine the data set graphically, we see that TISS better separates survivors from non-survivors. This impression is confirmed by the doubling of the separation of the median values for survivors and fatalities by TISS over NISS in both data sets. (TISS median fatalities - TISS median survivors = 29.9 and 29.0; NISS median fatalities NISS median survivors $=28.0$ and 27.8 in Hangzhou and Shenyang, respectively (Figures 1 and 2)).

The NISS and the TISS are different in approximately $98 \%$ of the incidents. Figures 3 and 4 show graphs of the NISS and the TISS against actual mortality. It is seen that, although the lines are generally increasing, the NISS scores are very choppy and have non-monotonic qualities. The NISS graphs show a nonlinear trend, confirming earlier research. Disparate TISS values have relevant mortality rates. A great number of NISS mortality rates were distributed to the right of the auxiliary line. TISS mortality rates were distributed at both sides of the auxiliary line.

A formal statistical analysis confirms the superior predictive power of TISS over NISS. A great number of measures examined were statistically sig- 

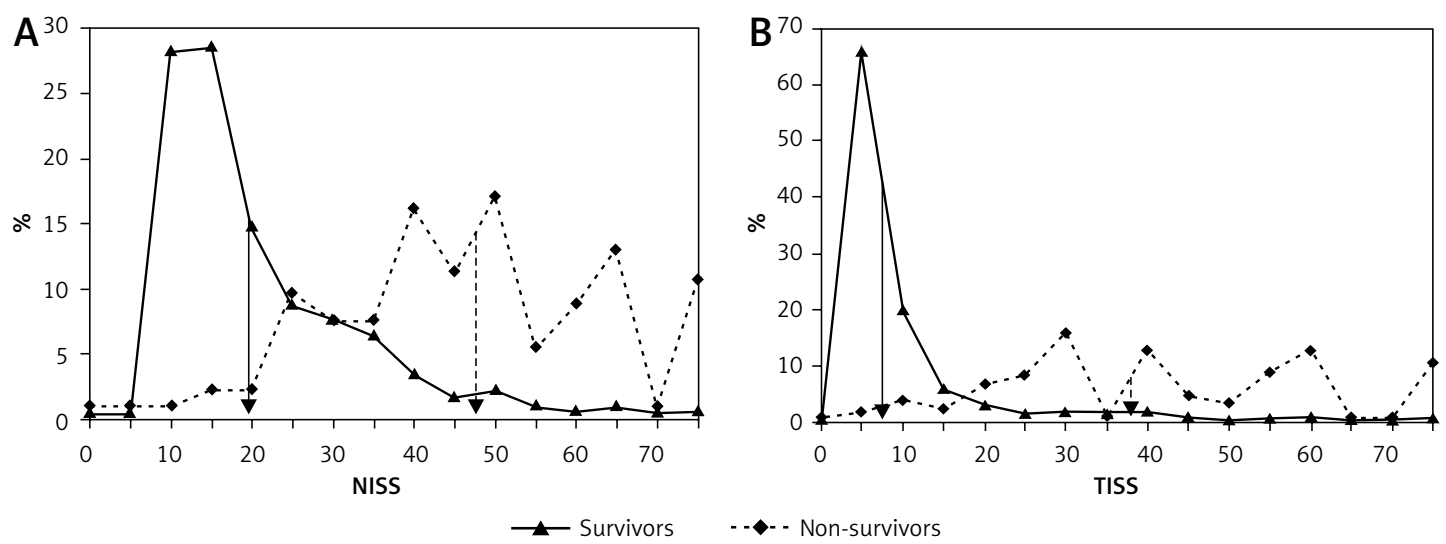

Figure 1. Hangzhou data set frequency distributions for survivors and mortalities as coded by NISS (A) and TISS (B). Arrows indicate median values, which are twice as widely separated by TISS. Solid curve and arrow = survivors, dashed line and arrow $=$ mortalities
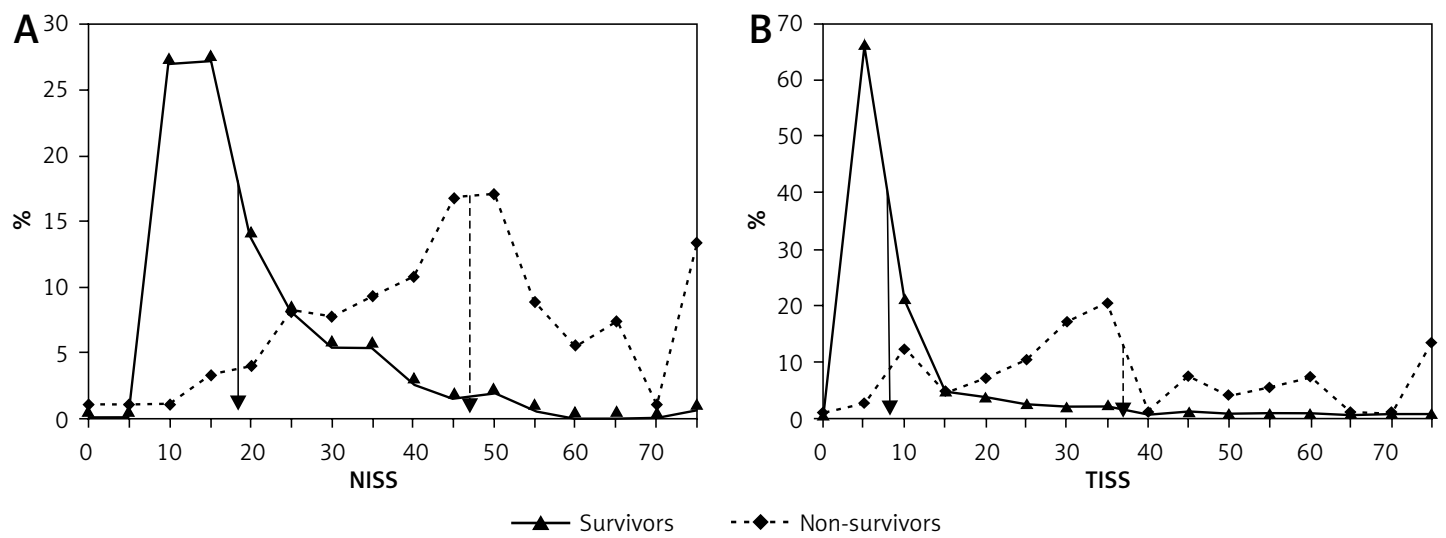

Figure 2. Shenyang data set frequency distributions for survivors and mortalities as coded by NISS (A) and TISS (B). Arrows indicate median values, which are twice as widely separated by TISS. Solid curve and arrow = survivors, dashed line and arrow $=$ mortalities
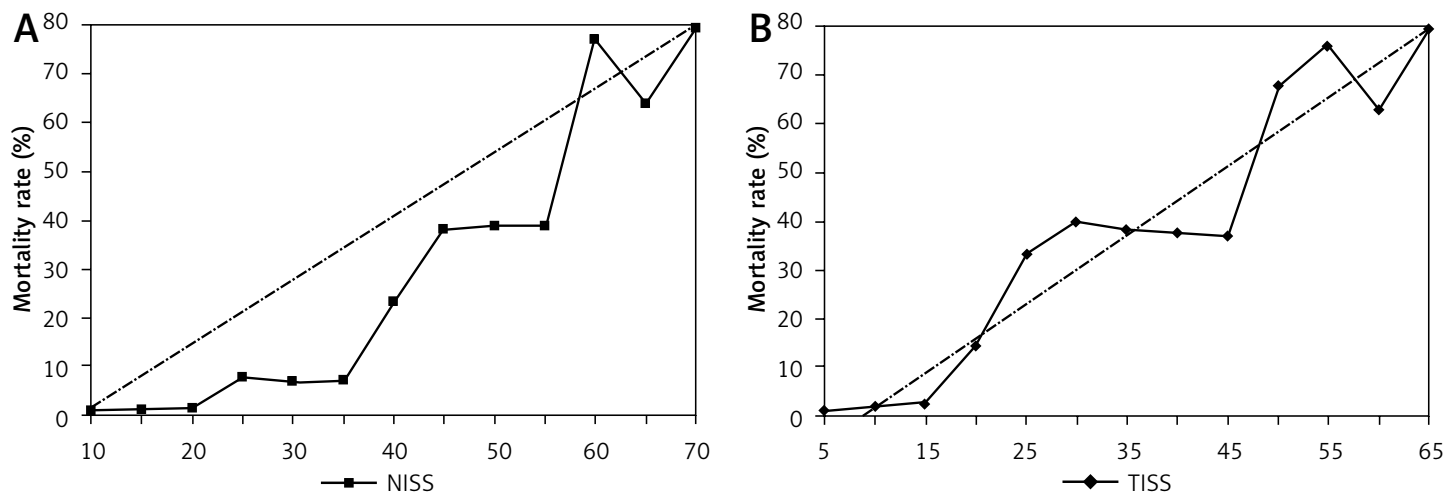

Figure 3. Hangzhou mortality rates for different NISS (A) and TISS (B) values. Solid curve $=$ NISS (A) and TISS (B). Dashed line is the auxiliary line that connects two points, where the curve crosses over the values of 10 and 75 on the horizontal axis respectively

nificantly better for TISS than for NISS: ROC curve areas, Hosmer Lemeshow statistics, and mean values (Table II). Only the misclassification rate is not statistically significantly improved under TISS.

\section{Discussion}

For even better estimation of injury severity, molecular biology and medicine will likely be ven- turing into cellular markers of injury magnitude, and these will be the "true" markers of injury severity [17]. Such markers as neuron-specific enolase (NSE), S100 calcium binding protein B and ubiquitin carboxy-terminal hydrolase-L1 have been proposed as potential markers for cell damage in the central nervous system. The level of $G$ protein related kinase-2 (GRK-2) could be an 

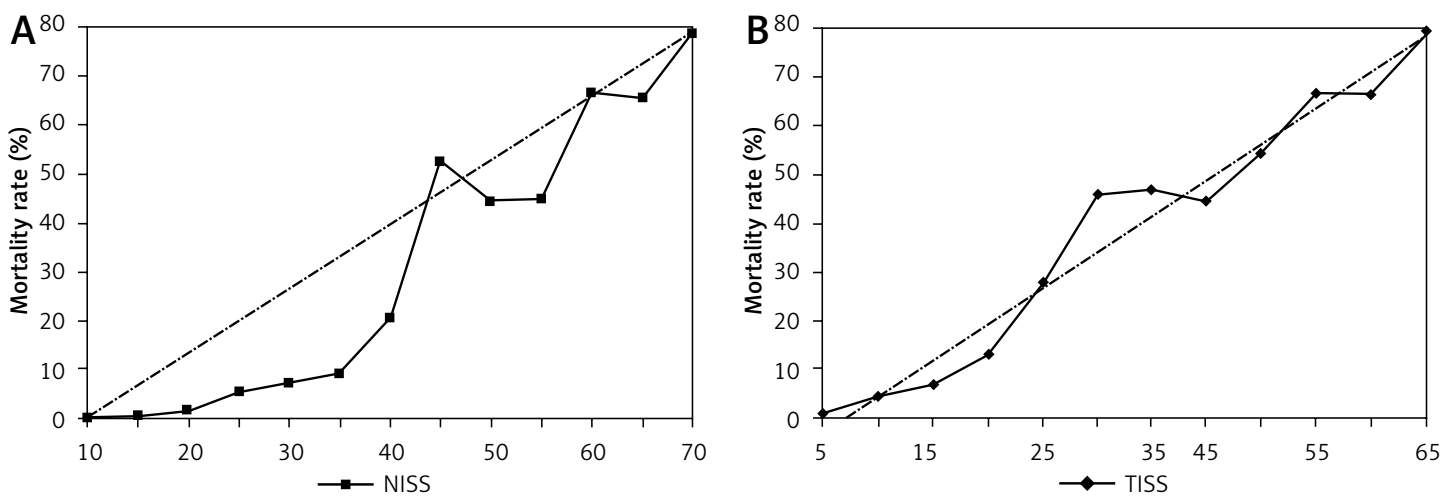

Figure 4. Shenyang mortality rates for different NISS (A) and TISS (B) values. Solid curve = NISS (A) and TISS (B). Dashed line is the auxiliary line that connects two points, where the curve crosses over the values of 10 and 75 on the horizontal axis respectively

Table II. Comparison of NISS and TISS in predicting mortality in two data sets

\begin{tabular}{|c|c|c|c|}
\hline Variable & NISS & TISS* & Value of $p$ \\
\hline \multicolumn{4}{|l|}{ Hangzhou: } \\
\hline Misclassification (\%) & 4.37 & 4.07 & 0.504 \\
\hline ROC & 0.929 & 0.949 & 0.002 \\
\hline ROC $95 \% \mathrm{Cl}$ & $0.916-0.943$ & $0.939-0.959$ & \\
\hline Hosmer-Lemeshow & $29.71(p<0.001)$ & $19.59(p=0.003)$ & \\
\hline \multicolumn{4}{|l|}{ Shenyang: } \\
\hline Misclassification (\%) & 4.58 & 4.34 & 0.595 \\
\hline ROC & 0.924 & 0.942 & 0.008 \\
\hline $\mathrm{ROC} 95 \% \mathrm{Cl}$ & $0.910-0.938$ & $0.931-0.954$ & \\
\hline Hosmer-Lemeshow & $33.49(p<0.001)$ & $21.19(p=0.002)$ & \\
\hline
\end{tabular}

indicator of $\beta$-adrenoreceptors (AR) blocker efficacy in patients with acute coronary syndrome [17-19]. Unfortunately, few markers have ever been found. Therefore the injury score system has been adopted for the evaluation of patients after trauma. The AIS severity score performs well as a measure of mortality, but that mortality is not the sole determinant of AIS severity. As AIS increased, the variability of survival also increased. This reflects the fact that when looking at mortality, there is such low mortality for all of the AIS 1 and 2 injuries that virtually no variability exists. Understandably, because other factors than mortality are involved in the AIS values, more complex injuries have wider variability [2]. Therefore this study ignores those patients injured with AIS severities of 1 and/or 2. Only data from those 181707 patients who sustained a single injury presented in the NTDB were analyzed [2]. There is a non-linear correlation between AIS severity and survival (and mortality). The data fit a quadratic function nearly perfectly. The mortalities for the patients with one injury of AIS severity on the ordinal scale 3, 4, 5, and 6 are 3.5\%, 14.6\%, 39.6\%, and $79.0 \%$, respectively. Their corresponding proportions approximately are $1: 4.2$ (14.6\% divided by $3.5 \%): 11.3: 22.6$. How can we transform a quadratic function relation into a more intuitive linear relation?

There are a variety of scoring methods. The capability of ISS to consider as many as three different injuries in its final outcome score represented a considerable advance over the earlier practice of summarizing a patient's injuries based on the single worst injury (maximum AIS). Today modern trauma databases routinely record all of the injuries that a patient sustains. It seemed likely to us that a more modern summary measure of trauma that could take advantage of this richer description of patients' injuries would more accurately predict outcome than the original ISS. The NISS, by contrast, simply considers the three most severe injuries that a patient has sustained and thus avoids this shortcoming of the traditional 
ISS. There are many articles [5-8] in the literature that have compared the NISS favorably to the ISS in predictive mortality, so this study will only compare the TISS with the NISS, and not the ISS.

But the ISS and NISS have an idiosyncrasy that impairs their predictive power. The ISS/NISS values for the patients with one injury of AIS severity on the ordinal scale are 3, 4, 5, and 6 in 9, 16, 25, and 75 , respectively. Their corresponding proportions approximately are $1: 1.8$ (16 divided by 9 ) : 2.8 : 8.6. Comparisons of the corresponding proportions between AIS and ISS/NISS were made using 1 divided by 1 is equal to $1,4.2 / 1.8=2.3,11.3 / 2.8=$ 4.0 , and 22.6/8.6 $=2.7$, respectively. The difference was at least one time more than if based on AIS 3. It showed that there are serious flaws in ISS/ NISS scoring.

The practical and statistical limitations of the ISS have been discussed in some papers. Although the ISS is scaled from 1 to 75 , it actually takes just 44 distinct values, and these values are not uniformly distributed. Statistical comparisons of the 44 ISS values are scaled from 1 to 75, $p=0.015$. Because of this, some have suggested that the ISS should not be treated as a continuous measure but rather as an ordinal scale, which is mathematically correct, if thoroughly ignored [16]. Although TISS actually takes just 21 distinct values, these values are uniformly distributed $(p=0.060)$. The TISS values for the patients with one injury of AIS severity on the ordinal scale are 3, 4, 5, and 6 in $3,9,25$, and 75 , respectively. Their corresponding proportions approximately are $1: 3.0$ (9 divided by $3): 8.3: 25.0$. Their proportions are roughly equal to the mortality of each AIS code.

Some studies have shown differences for nonunique values of the ISS and the NISS respectively. The AIS triplets that produce identical ISS and NISS scores have markedly different mortality rates [13-16]. For instance, an ISS of 27 is possible in two groups of patients where $(5,1,1)$ had a $35.25 \%$ mortality rate in comparison with $11.31 \%$ in $(3,3,3)$, representing a $23.94 \%$ difference in mortality even though their ISS scores are identical [14]. The TISS adjust its $(5,1,1)$ mortality rate to $35.25 \% \times(3+3+3) /(25+0+0)=12.69 \%$, and comparing mortality rates AIS triplets $(5,1,1)$ and AIS triplets $(3,3,3)$ again, $p=0.069$. Similarly, several ISS values coming from different trauma lead to a change in the statistical significance of mortality. However, TISS lessens significant differences in mortality rates compared to the AIS triplets which produce identical scores. This shows that ISS scores with a higher AIS value are more likely lead to mortality, and a quadratic function of the AIS value cannot be solved.

The TISS prediction of mortality is based solely on the anatomic information specified by a pa- tient's AIS injury descriptors. We believe that part of the value of an injury summary score is that it can be calculated by clinicians. The popularity of ISS/NISS has stemmed in some measure from its ease of computation. This is a retrospective, non-concurrent cohort study that compares TISS with NISS values calculated at the time of discharge. A concurrent cohort study would presumably yield identical results, but would be of interest to further verify our results.

The study is only based on two centers' experience, and therefore the findings may not be fully generalizable. Additionally, it is possible that our cohort does not include those patients injured with AIS severities of 1 and/or 2 . As we all know, all injury-based systems such as the AIS are limited by the "knowns" and the "unknowns". This TISS system, in addition to "ignoring" a certain portion of injured patients, is also dependent on accurate and prompt injury identification. It may affect the outcome. While trauma registrars, all fully trained in data collection and coding, are responsible for data collection, the interreliability of AIS coding between hospitals is a different possible human factor. The scope of trauma service provided by different hospitals is different; the trauma type and severity can also be different, and this will directly affect the performance and outcome. The misclassification rate is not statistically significantly improved under TISS in the study.

In conclusion, although the calculation of TISS is itself so complicated that a computer is required, the result is simple. This corresponds to the basic principles of the scoring system: that it is simple and easy to remember. Furthermore, TISS better predicts mortality than NISS. The TISS might be better than ISS/NISS as a standard summary measure of human trauma.

\section{Acknowledgments}

The present study was supported by the Scientific Research Fund of Hangzhou Health Department (No. 2010A013) and the Scientific Research Fund of Science and Technology Department of Hangzhou (No. 20120533Q22), China.

\section{Conflict of interest}

The authors declare no conflict of interest.

\section{References}

1. Association for the Advancement of Automotive Medicine (2008). The Abbreviated Injury Scale 2005 update 2008. Barrington, IL.

2. Gennarelli TA, Wodzin E (eds.). The Abbreviated Injury Scale 2005. Des Plaines, IL: Association for the Advancement of Automotive Medicine (2005).

3. Baker SP, O'Neill B, Haddon W, et al. The injury severity score: a method for describing patients with multiple 
injuries and evaluating emergency care. J Trauma 1974 14: 187-96.

4. Osler T, Baker SP, Long W. A modification of the injury severity score that both improves accuracy and simplifies scoring. J Trauma 1997; 43: 922-6.

5. Sullivan T, Haider A, DiRusso SM, et al. Prediction of mortality in pediatric trauma patients: new injury severity score outperforms injury severity score in the severely injured. J Trauma 2003; 55: 1083-7.

6. Lavoie A, Moore L, LeSage N, et al. The new injury severity score: a more accurate predictor of in-hospital mortality than the injury severity score. J Trauma 2004; 56: 1312-20.

7. Frankema SPG, Steyerberg EW, Edwards MJR, et al. Comparison of current injury scales for survival chance estimation: an evaluation comparing the predictive performance of the ISS, NISS, and AP scores in a Dutch local trauma registration. J Trauma 2005; 58: 596-604.

8. Hu Y, Tang Y, Yuan Y, et al. Trauma evaluation of patients with chest injury in the 2008 Earthquake of Wenchuan, Sechuan, China. World J Surg 2010; 34: 728-32.

9. Tay SY, Sloan EP, Zun L, et al. Comparison of the new injury severity score and the injury severity score. J Trauma 2004; 56: 162-4.

10. Bulut M, Koksal O, Korkmaz A, et al. Childhood falls: characteristics, outcome, and comparison of the injury severity score and new injury severity score. Emerg Med J 2006; 23: 540-5.

11. Stephenson SCR, Langley JD, Civil ID. Comparing measures of injury severity for use with large databases. J Trauma 2002; 53: 326-32.

12. Tamim H, Al Hazzouri ZA, Mahfoud Z, et al. The injury severity score or the new injury severity score for predicting mortality, intensive care unit admission and length of hospital stay: experience from a university hospital in a developing country. Injury 2008; 39: 115-20.

13. Russel R, Halcomb E, Caldwel E, et al. Differences in mortality predictions between injury severity score triplets: a significant flaw. J Trauma 2004; 56: 1321-4.

14. Kilgo PD, Meredith JW, Hensberry R, et al. A note on the disjointed nature of the injury severity score. J Trauma 2004; 57: 479-87.

15. Aharonson-Daniel L, Giveon A, Stein M, et al. Different AIS triplets: different mortality predictions in identical ISS and NISS. J Trauma 2006; 61: 711-7.

16. Krischer JP. Indexes of severity: underlying concepts. Health Serv Res 1976; 11: 143-57.

17. Bojic T, Sudar E, Mikhailidis D, et al. The role of $G$ protein coupled receptor kinases in neurocardiovascular pathophysiology. Arch Med Sci 2012; 8: 970-7.

18. Zetterberg H, Smith DH, Blennow K. Biomarkers of mild traumatic brain injury in cerebrospinal fluid and blood. Nat Rev Neurol 2013; 9: 201-10.

19. Zakaria S, Youssef $M$, Moussa $M$, et al. Value of alpha-smooth muscle actin and glial fibrillary acidic protein in predicting early hepatic fibrosis in chronic hepatitis C virus infection. Arch Med Sci 2010; 6: 356-65. 